\title{
Deep Water Dredging and Maintenance in the Offshore Industry
}

\author{
Ellen Stuifbergen MSc, \\ The Netherlands
}

Ellen Stuifbergen, Sales Manager Software Systems at RESON B.V.

Topic: Innovations in acquisition techniques

\section{INTRODUCTION}

The demand for high accuracy operations and positioning in bathymetric surveys is a current requirement in deep water dredging and construction projects. It is expected to become even more important in the future.

Removal and relocation of soil at depths as deep as 2,500m is a challenge. Reef Subsea Dredging \& Excavation, formerly known as Scanmudring located in Norway, is involved in deep water projects. Their deep water hydraulic excavator is especially designed for those projects.

Operated from the vessel the excavator crawls its way over the seabed. The excavator removes soil and places soil around the legs of rigs with high precision. It is also used to maintain offshore facilities or to tear down the object in an environmental safe way.

Depth and required accuracy puts a lot of strain on both the subsea equipment and the operator.

Reef Subsea Dredging \& Excavation requested RESON to assist in the development of the monitoring system.

Since this excavator is operated and controlled from the surface, it is very important that the operator monitors the system in real time and can trust the visualized information. Furthermore the software has to be intuitive and easy to use.

This paper describes the challenges of deep water operations and the different tools used to make high accuracy deep water dredging and construction a reality. The system has been operational since February 2012 when the first sea trials proved successful.

\section{VARIETY OF UNDERWATER OPERATIONS}

The Deep Water Excavator is used for a variety of underwater operations: Seabed preparation, material removal, trench dredging, soil removal from templates, pipes or other objects. The realisation of the project is complex as objects in the work area should not be damaged during dredging / construction operation. Damage could result in costly repairs or environmental damage.

A stable platform is a requirement for accurate operations. Standing on the seafloor the system has a high stability and can be used for precise handling of equipment and objects. For this reason the excavator is equipped with different sensors making accurate operation in deep water possible.

\section{POSITIONING OF THE EXCAVATOR}

High accuracy is not only important in shallow water bathymetric surveys, but also in deep water dredging and construction. It is expected to become even more important in the future as more work is carried out in deeper waters. Challenges at deep water are obviously the water depth, but also the accuracy required at that depth. This is of course, not only a challenge for the equipment and dredge tools, but also for the positioning and monitoring software and the operator. The operator is at the surface and he will fully rely on the information that is visualized through the monitoring software.

In this case RESON software PDS2000 is used to monitor the system operations. An USBL positioning system is used for the initial excavator positioning during the descent and touch down. USBL positioning accuracy has improved a lot in the last decade, but still not always accurate or stable enough to perform the operations at the high accuracy required. So once the excavator is near its operation location, the excavator position is recomputed using known reference points on the seabed. 
This provides a better position than the USBL position alone. This new recomputed position will then be used in the software as a fixed reference position.

Various sensors are placed on the excavator to monitor changes in the position and height of the excavator. The excavator is equipped with accurate inclino sensors. The sensors are used to measure the excavator's boom, stick and bucket angles. Both inclinometer size and accuracy are equally important. RESON has carried out performance tests of different inclinometer brands to be able to supply the most suitable sensor. Other sensors as depth sensor, height sensors, roll/pitch and heading sensors complete the sensor configuration.

\section{MONITORING THE OPERATION}

The positioning sensors together with PDS2000 software and additional tools such as SeaBat Forward Looking systems and Multibeam Echosounders are used to monitor and improve accuracy of deep water operations.

The various sensors monitor height, roll and pitch of the excavator. These values are important to the operator, because they are an indication of the system stability. For example a weak seabed may cause the excavator to shift / tilt and reposition of the excavator may be required. The operator will see the position change in the 2D and 3D views. Additionally alarms can be generated when a sensor passes a certain threshold value.

As Figure 1 shows the software visualizes objects on the seabed as 3D shapes in the $3 \mathrm{D}$ view and in the topview. Alarms will be generated when the excavator is too close to one or more of the objects. Alarms can vary from a general warning to a signal which is generated that stops the excavator immediately to avoid collision.
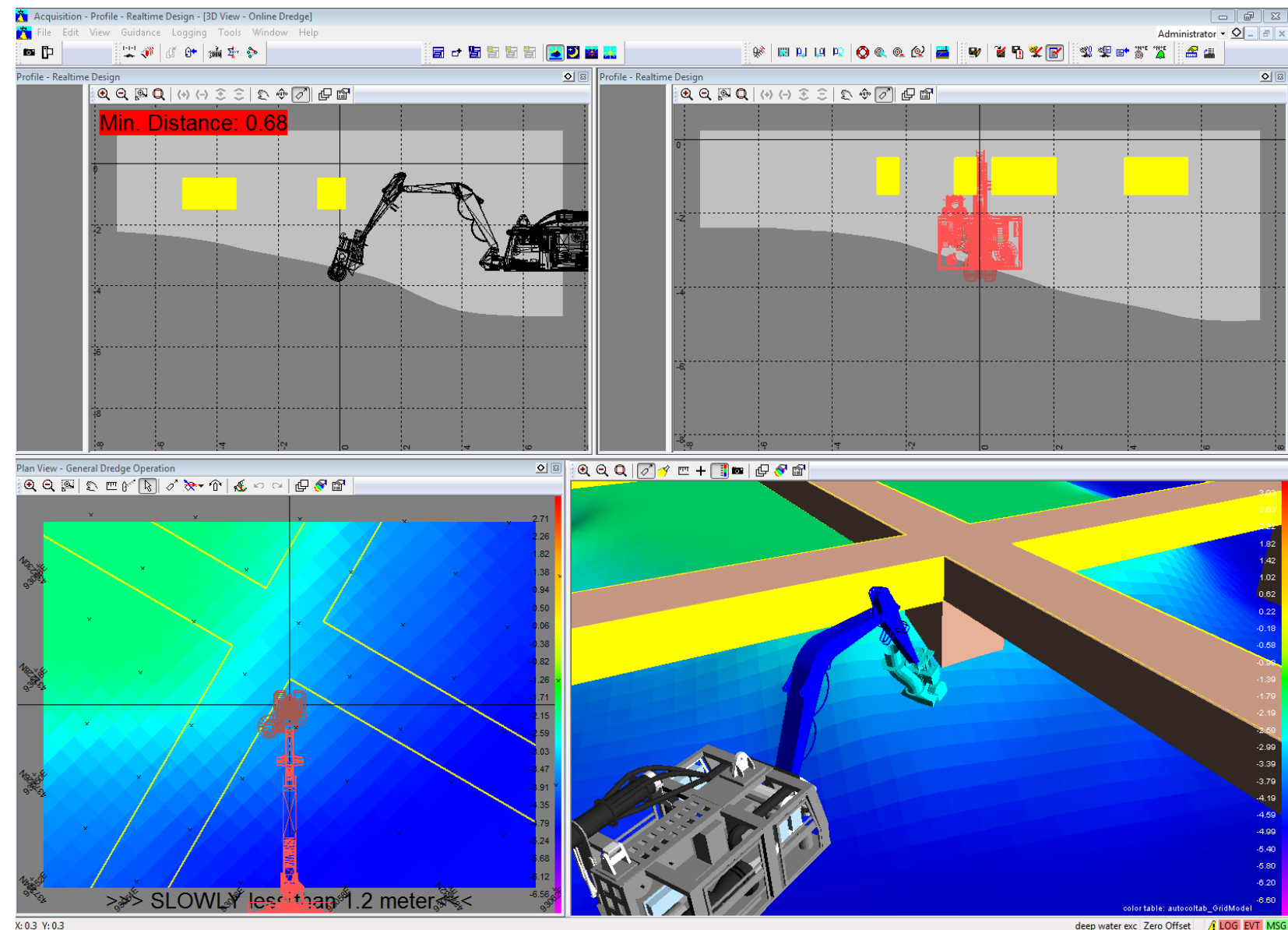

Figure 1. Simplified example of object avoidance 
An inspection ROV can also be used to monitor the excavator activities. To visualise and monitor both the ROV and excavator in the same 3D view, the ROV can simply be added to the PDS2000 configuration. Figure 2 shows both the excavator and ROV in the same 3D view.

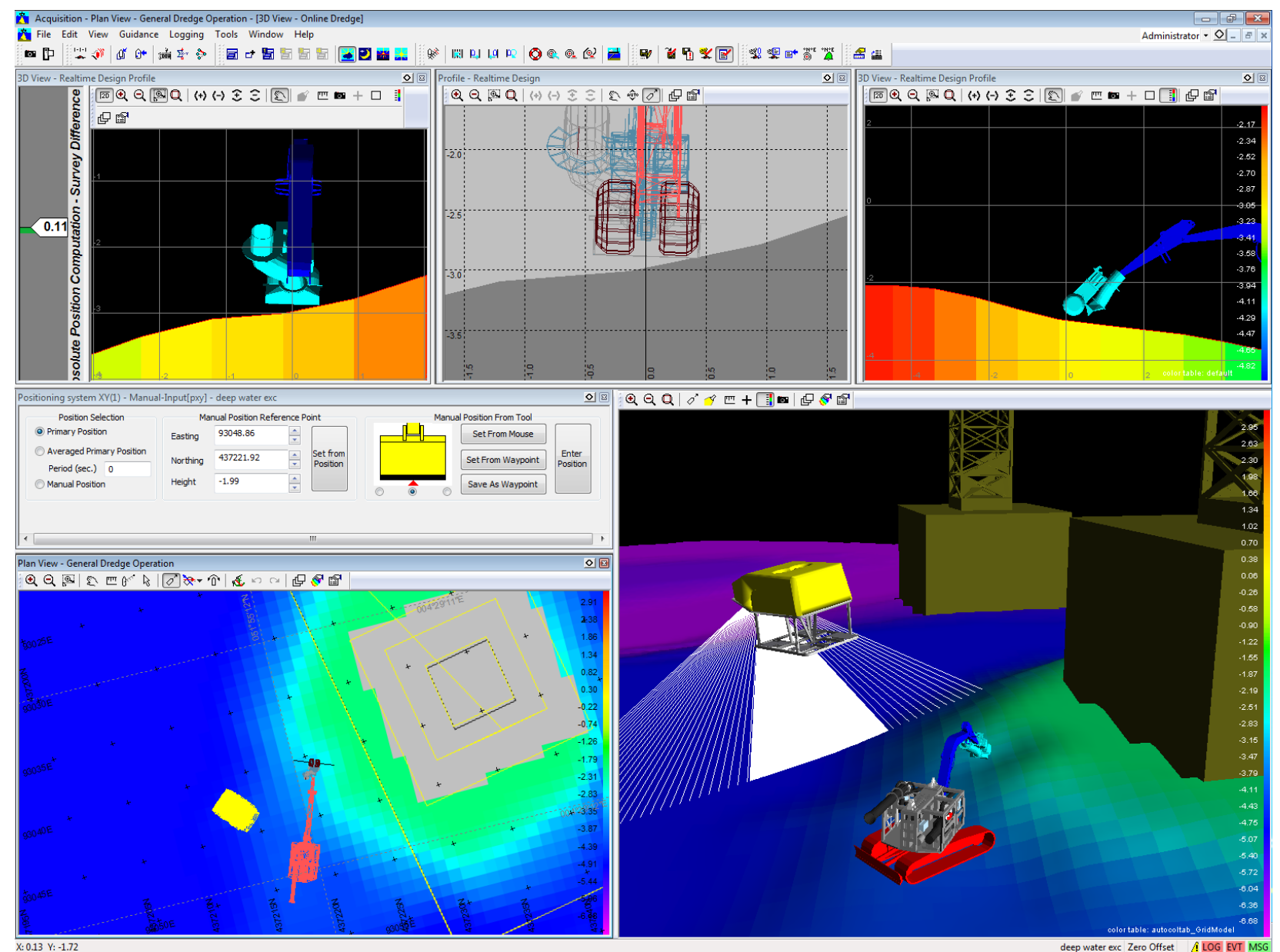

Figure 2. Excavator and ROV

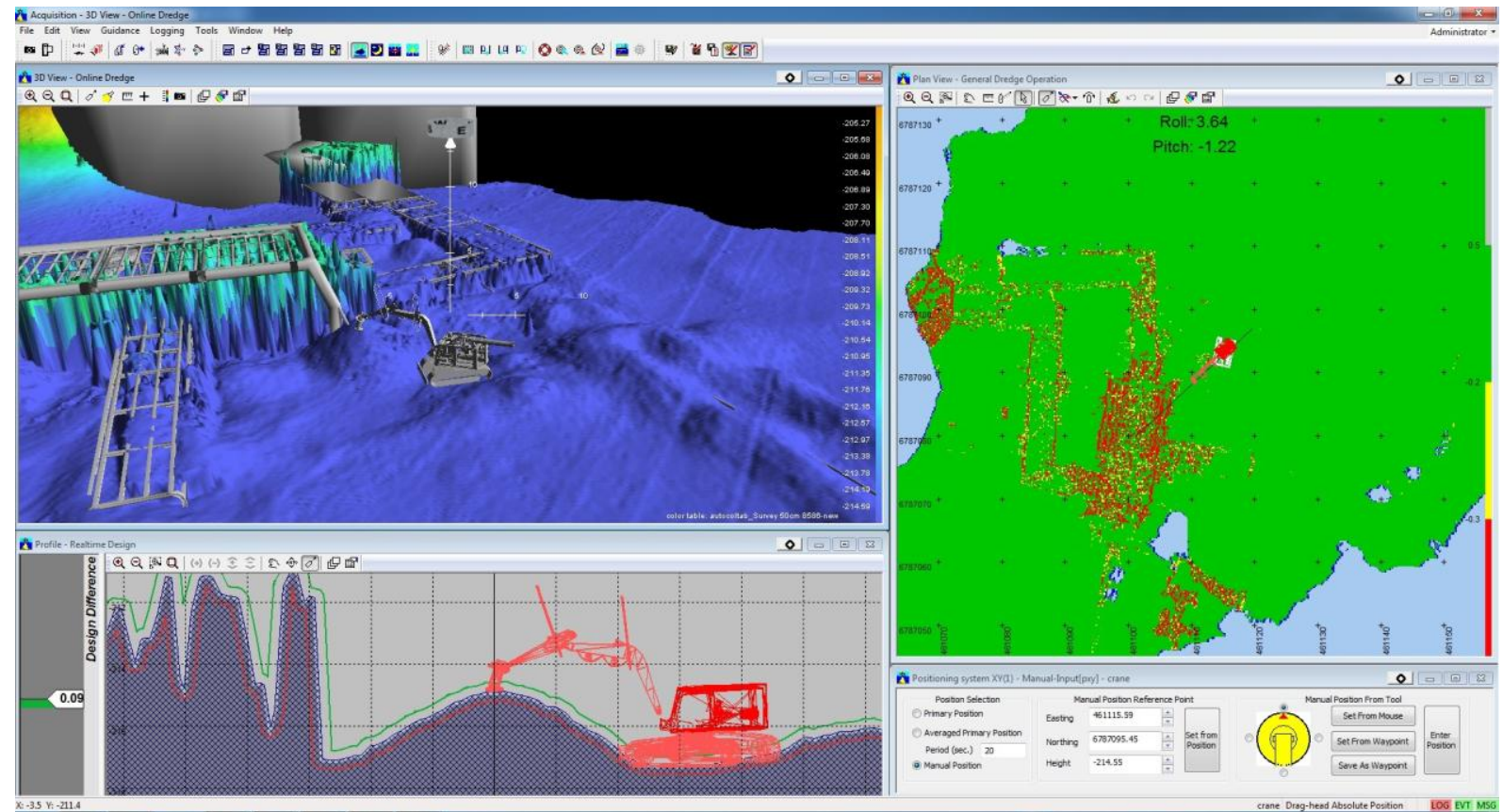

Figure 3. Excavator in operation at a depth of $240 \mathrm{~m}$ 
When visibility is good the camera on the excavator is used, but when visibility degrades the operator will have to rely on the Forward Looking Sonar (FLS) and PDS2000 monitoring software. The forward looking sonar SeaBat 7128 shows the details of the seafloor and objects in high resolution (Figure 3 and 4) The FLS image will also show the excavator tool movements and thus helps the operator in poor visibility situations.

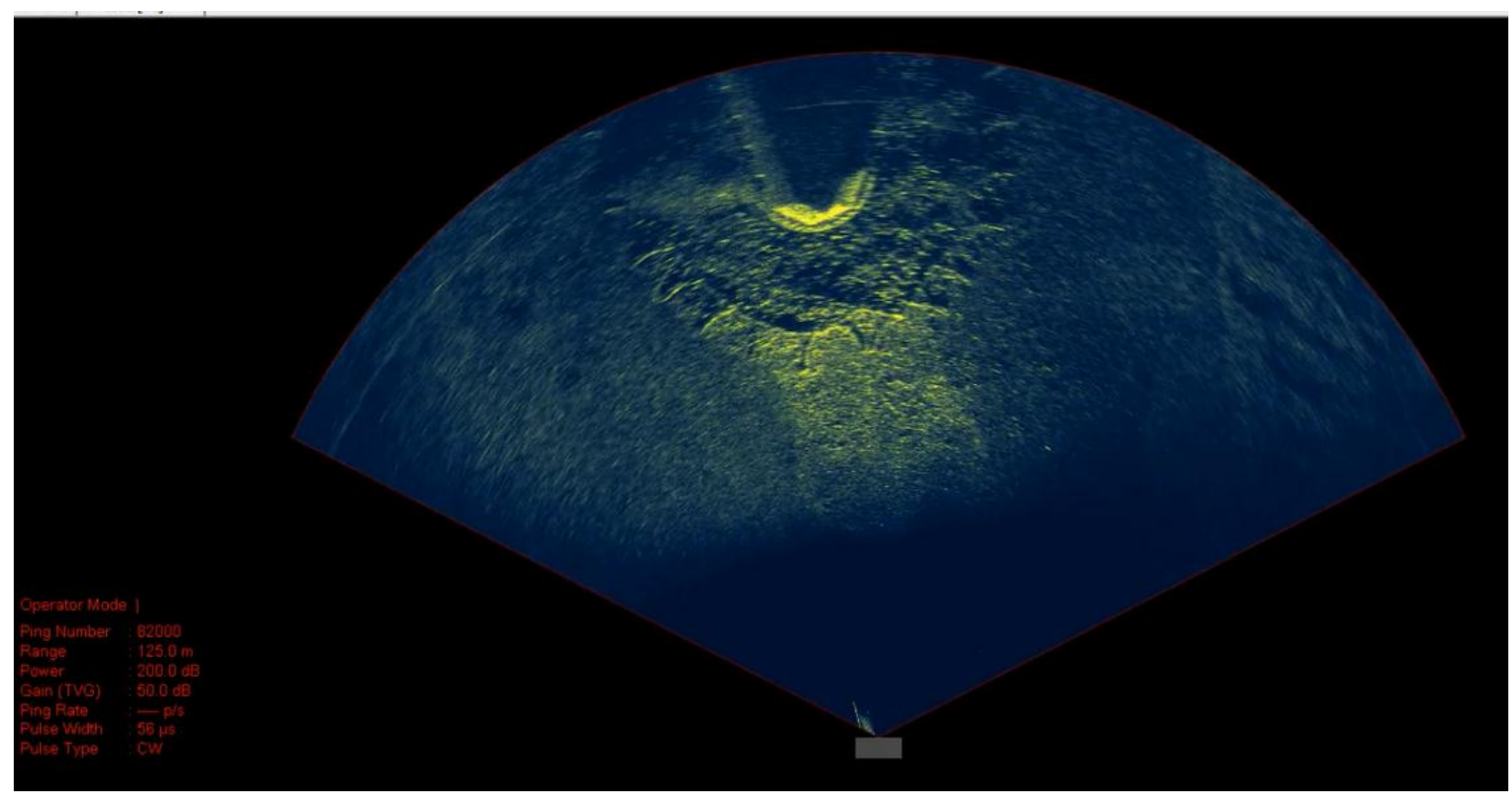

\section{Figure 4. Forward looking SeaBat 7128 at a pillar at a range of $125 \mathrm{~m}$. (400 Khz)}

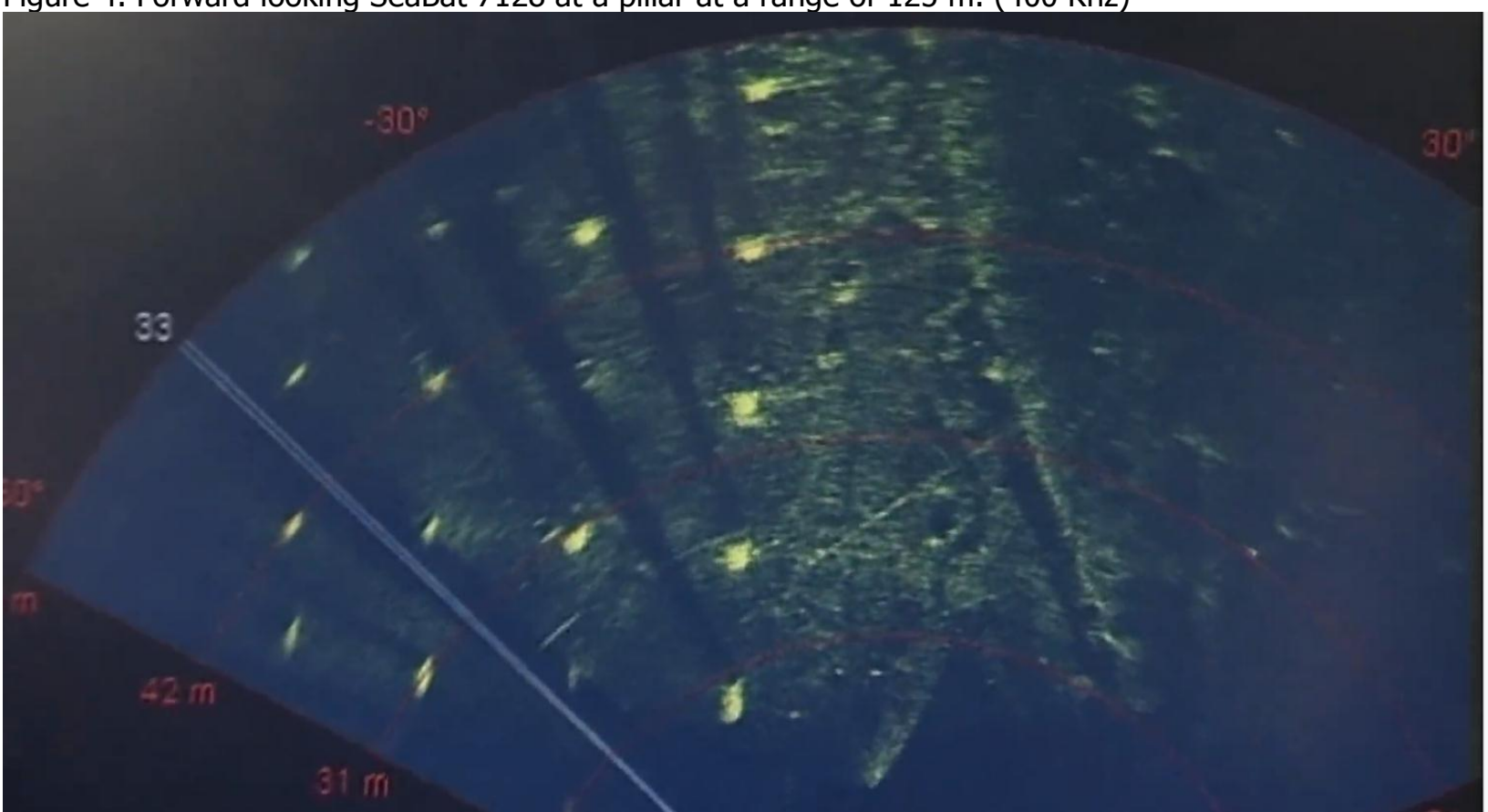

Figure 5. SeaBat 7128 forward looking at piles at a distance of $50 \mathrm{~m} .(400 \mathrm{Khz})$ 
During dredge operation PDS2000 monitors and updates the dredge progress in an As-Dredged gridmodel using the information of the sensors installed on crawler and excavator. A multibeam survey can be carried out during or after dredge activities to verify the work carried out. The multibeam data is used to update the As-Dredged Gridmodel

It is also possible to monitor the dredging operation while dredging. Figures 6 and 7 show these results.
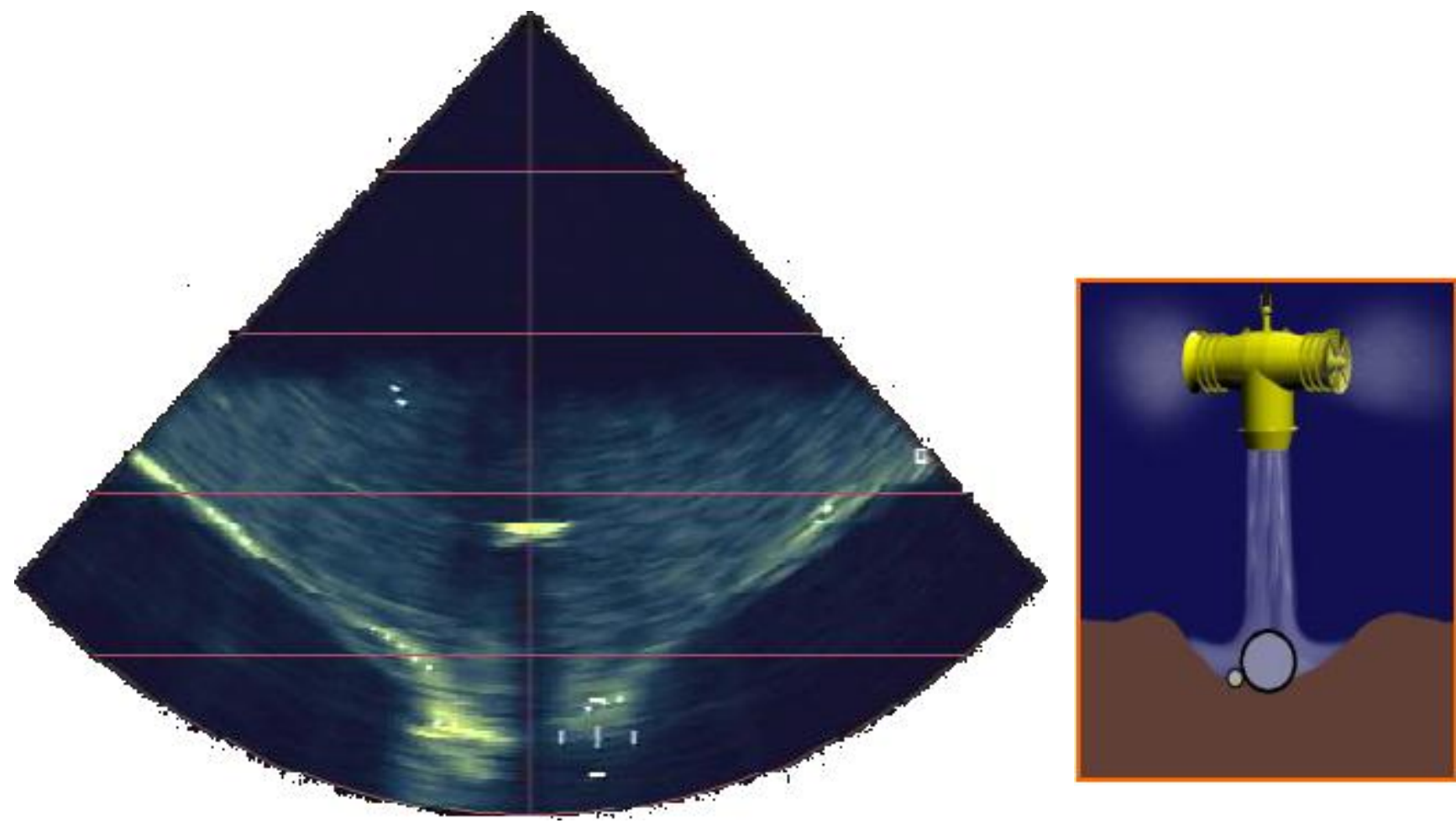

Figure 6. Monitoring the dredge activities with a Multibeam FLS system
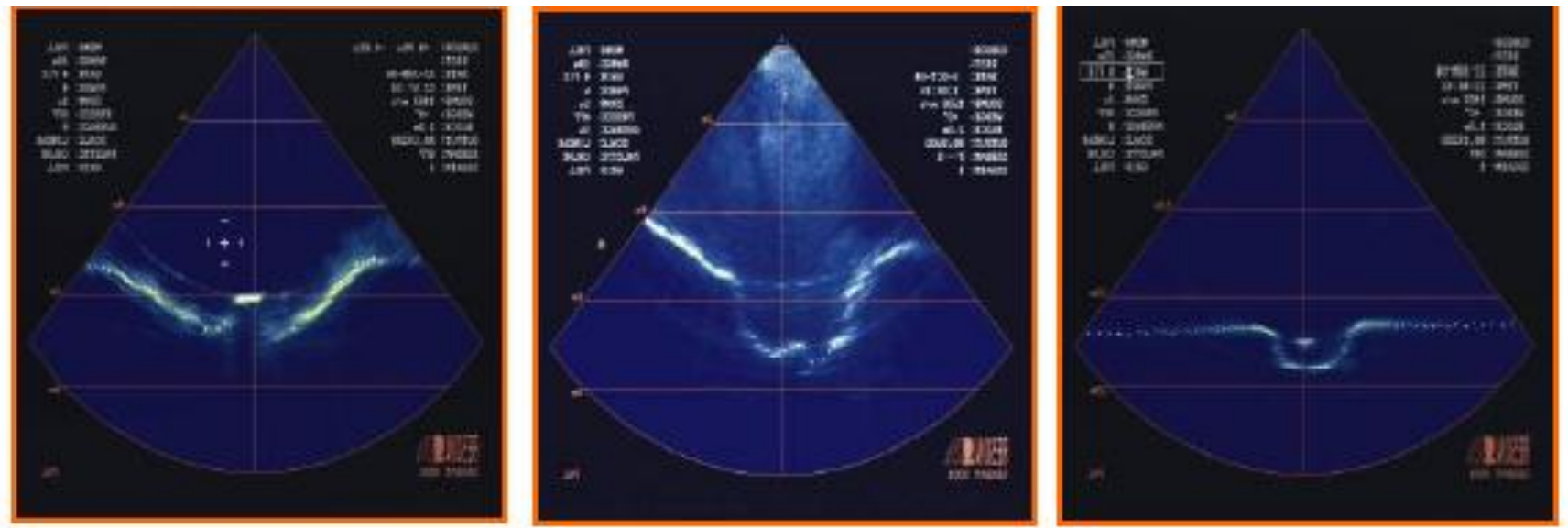

Figure 7. Monitoring the dredge activities with a Multibeam FLS system

\section{CONCLUSION}

The demand for high accuracy information in deep water dredge and construction projects is very important and will become even more important in the future. The use of monitoring software with different sensors, such as USBL, inclinometers and the use of existing known positions on the seabed, allows work to be carried out quickly and safely. This is thanks to the real time excavator visualization in $2 \mathrm{D}$ and $3 \mathrm{D}$ views. User configurable alarms provide an extra level of security. Acoustic imaging systems such as FLS help the operator in poor visibility conditions and multibeam echosounders provide verification during and after the dredge operation. 
The combination of different sensors, acoustic positioning systems and reliable monitoring software makes it possible to achieve and work with higher accuracy in deep water.

\section{BIOGRAPHIES}

Ellen Stuifbergen, Sales Manager Software Systems at RESON B.V.

Started as geodetic surveyor at NeSA, a RACAL Company in 1993 and graduated as MSc Geodesy at the Technical University in Delft.

RESON is manufacturer of SeaBat Multibeam and Forward Looking Systems as well as the Software PDS2000. Next to that RESON also manufactures and supplies sensors to measure the movements of the various dredge tools. A combination of these tools are used to improve the monitoring of the deep water operations as well as the accuracy of the operation

CONTACT DETAILS (of corresponding author only)

Ellen Stuifbergen

RESON B.V.

Stuttgartstraat 42-44

3047 AS Rotterdam

The Netherlands

Tel.: +31102451500

Fax: + 31102451555

Email: ellen.stuifbergen@reson.com

Web site: www.reson.com

Twitter account: @PDS2000 